\title{
Etude multi-échelles des contraintes résiduelles de fabrication dans un matériau composite méso-structuré
}

\author{
A multi-scale study of residual cure stresses \\ in a meso-structured composite material \\ Emmanuel Lacoste ${ }^{1}$, Frédéric Jacquemin ${ }^{1}$ et Sylvain Fréour ${ }^{1}$ \\ 1 : Institut de Recherche en Génie Civil et Mécanique (UMR CNRS 6183), \\ LUNAM Université, Université de Nantes, Ecole Centrale de Nantes \\ 37, Bd de l'Université, BP 406, 44602 Saint-Nazaire, France \\ e-mail : emmanuel.lacoste@univ-nantes.fr, frederic.jacquemin@univ-nantes.fr, sylvain.freour@univ-nantes.fr
}

\begin{abstract}
Résumé
Cet article traite du calcul des contraintes résiduelles de cuisson dans le Hextool ${ }^{\circledR}$ [1], un matériau composite carbone organique, qui se présente comme un mat à renforts mésoscopiques courts. Une modélisation multiéchelles de son comportement mécanique a été effectuée à l'aide d'un modèle d'homogénéisation « à champ moyen » (modèle autocohérent d'Eshelby-Kröner [2,3]). Une méthode en deux étapes est utilisée, afin de représenter la microstructure des renforts unidirectionnels, puis la mésostructure du pli Hextool, et permet de calculer les propriétés effectives du matériau, ainsi que les contraintes locales (résiduelles et de service). Des données expérimentales ont permis d'établir la cinétique de cuisson de la résine, et de modéliser son durcissement, qui est pris en compte de façon incrémentale. Les résultats indiquent que les contraintes résiduelles impactent fortement la résistance du matériau en service.
\end{abstract}

\begin{abstract}
This paper is dedicated to the computation of the residual cure stresses in the Hextool® [1], a carbon-organic composite material, which is represented as a matt made of short mesoscopic reinforcements. A multi-scale simulation of its mechanical behaviour is carried out with a "mean-field" homogenization model (Eshelby-Kröner's self-consistent model [2,3]). A two-steps method is used in order to represent the microstructure of the unidirectional reinforcements, then the mesostructure of the Hextool ply, and enables one to compute the effective properties of the material and the (residual and service) local stresses. Some experimental data are used to establish the cure kinetics of the material, and the hardening phenomenon, which is taken into account incrementally. The results indicate that the residual stresses have a heavy impact on the resistance of the material in service life.
\end{abstract}

Mots Clés : composites, contraintes résiduelles, fabrication, multiéchelles

Keywords : composites, residual stresses, processing, multiscale

\section{Introduction}

\subsection{Problématique}

La question des contraintes résiduelles et locales est une problématique fondamentale pour qui s'intéresse à la tenue en service des matériaux composites. Du fait de l'hétérogénéité des propriétés mécaniques du matériau à l'échelle microscopique, des champs de contraintes complexes peuvent apparaître. En particulier, durant le procédé de cuisson du matériau, le retrait thermochimique de la résine génère des auto-contraintes considérables dans les constituants, qui jouent de façon importante sur le comportement ultérieur du matériau. L'estimation de ces contraintes est compliquée par la forte évolution des propriétés mécaniques de la résine au cours du procédé de fabrication (voir la revue bibliographique très complète de Parlevliet et al. [4-6]), qui nécessite de recourir à un traitement incrémental du problème, et donc à un grand nombre de calculs. Un problème corollaire est le calcul des propriétés mécaniques du matériau (homogénéisation), qui intervient notamment lorsqu'il y a couplage entre les effets structurels dans la pièce et les comportements locaux dans le matériau. 


\subsection{Revue des modèles}

Les contraintes résiduelles microscopiques ou mésoscopiques peuvent être évaluées à l'aide de diverses méthodes expérimentales [5], ou via l'une des approches de simulation existantes. Ainsi, pour les matériaux à microstructure très irrégulière, l'approche asymptotique ou « à champ complet », qui repose sur une modélisation directe du Volume Elémentaire Représentatif (V.E.R.) par la méthode des éléments finis, donne de très bon résultats mais au détriment du coût de calcul (difficultés dans la génération de la microstructure et la résolution du problème). L'approche périodique (méthode $\mathrm{FE}^{2}$ par exemple) permet de simplifier largement le problème en assimilant la microstructure à un réseau périodique, mais cette description n'est réaliste que pour certains matériaux à microstructure régulière. Enfin, la dernière approche, couramment utilisée pour ce type de problème, est l'approche «à champ moyen », dans laquelle le V.E.R. est décrit de façon statistique et où l'arrangement géométrique des inclusions n'est pas pris en compte. Cette méthode permet une très grande flexibilité dans la description de la microstructure ainsi qu'un coût de calcul très faible, mais au détriment de la qualité des résultats, puisqu'on n'obtient qu'une valeur moyenne des états locaux. Dans cette étude, on mettra à profit l'efficience de l'approche à champ moyen afin de donner une première estimation des contraintes multi-échelles dans le Hextool, qui prend en compte l'évolution des propriétés mécaniques de la résine durant le procédé de fabrication.

\subsection{L'approximation d'Eshelby}

Les modèles d'homogénéisation, et particulièrement les modèles à champ moyen, reposent sur une représentation du matériau à deux échelles bien séparées : l'échelle macroscopique (identifiée par un exposant ${ }^{\mathrm{I}}$ ) pour laquelle le matériau est supposé homogène, et l'échelle locale ou microscopique (identifiée par un exposant ${ }^{i}$ ), où le matériau est supposé hétérogène. Ici, on supposera que le comportement mécanique du matériau est décrit par une loi thermo-chimio-élastique instantanée, représentée par l'équation suivante :

$$
\dot{\sigma}^{\mathbf{k}}=\mathbf{L}^{\mathbf{k}}:\left(\dot{\varepsilon}^{\mathrm{k}}-\alpha^{\mathrm{k}} \dot{\mathrm{T}}-\eta^{\mathrm{k}} \dot{\chi}\right), \text { avec } \mathrm{k}=\{\mathrm{i}, \mathrm{I}\}
$$

où les vitesses de contraintes, de déformations, de chauffe et de réticulation sont représentées respectivement par $\dot{\boldsymbol{\sigma}}, \dot{\boldsymbol{\varepsilon}}$, $\dot{\mathbf{T}}$ et $\dot{\boldsymbol{\chi}}$. Les propriétés mécaniques instantanées du matériau sont représentées par les tenseurs de rigidité $\mathbf{L}$, de dilatation thermique $\boldsymbol{\alpha}$ (CDT) et chimique $\boldsymbol{\eta}$ (CDC). Pour l'approche à champ moyen, le matériau est représenté à l'échelle locale comme un milieu infini dans lequel baignent des inclusions de forme ellipsoïdale. Les relations d'homogénéisation sont établies via des relations de moyenne volumique sur les états mécaniques dans le V.E.R. [7] :

$$
\boldsymbol{\varepsilon}^{\mathrm{I}}=\left\langle\boldsymbol{\varepsilon}^{\mathrm{i}}\right\rangle \text { et } \boldsymbol{\sigma}^{\mathbf{I}}=\left\langle\boldsymbol{\sigma}^{\mathbf{i}}\right\rangle
$$

Les relations de localisation sont établies à partir des travaux d'Eshelby sur le comportement d'une inclusion ellipsoïdale noyée dans un milieu homogène infini, qui ont permis à Hill d'établir la relation suivante :

$$
\sigma^{\mathrm{I}}-\sigma^{\mathrm{i}}=-\mathbf{L}^{*}:\left(\varepsilon^{\mathrm{I}}-\varepsilon^{\mathrm{i}}\right)
$$

Dans cette expression, le tenseur des contraintes de Hill $\mathbf{L}^{*}$ est calculé à partir des propriétés élastiques du milieu choisi comme phase mère (ou milieu ambiant). Il prend en compte la morphologie des inclusions, c'est à dire leur orientation et leurs rapports de forme, ce qui est un progrès par rapport aux modèles de type «lois des mélanges » (modèles de Voigt ou de Reuss, par exemple). Par ailleurs, le choix du milieu ambiant est aussi une hypothèse fondamentale des modèles utilisés. Ainsi, dans le cas de modèle de Mori-Tanaka [8], très utilisé pour les matériaux présentant des taux de renforts faibles, on choisit la résine comme milieu ambiant. Pour les 
matériaux dans lesquels aucune phase principale ne se démarque (par exemple les métaux polycristallins), on préfère utiliser le modèle autocohérent, dans lequel on attribue au milieu ambiant les mêmes propriétés que le matériau à l'échelle macroscopique [2,9]. Les modèles basés sur l'inclusion d'Eshelby proposent donc une approximation des champs mécaniques locaux : les interactions directes entre inclusions ne sont pas prises en compte, et la notion de phase continue (ici, la résine) est gommée. En contrepartie de cette approximation, la vitesse de calcul est très importante par rapport à celle des approches à champ complet.

\section{Description de la modélisation}

\subsection{Structure du matériau et méthode de transition d'échelles}

Cette étude est centrée sur l'étude d'un matériau composite développé par Hexcel Composites pour la conception de pièces composites complexes [1]. Ce matériau est formé par des renforts unidirectionnels (UD) de fibres de carbone AS4 et de résine bismaléimide M61, avec un taux volumique de fibres de $53 \%$ (global). Ces renforts sont coupés en bandes de dimensions $50 \times 8 \times 0,15 \mathrm{~mm}$, disposées aléatoirement dans le plan du pli (Fig. 1). Le Hextool a donc un comportement isotrope plan, caractérisé par un faible CDT (proche de celui des pièces composites classiques), une excellente tenue en température (jusqu'à plus de $220^{\circ} \mathrm{C}$ ), et une bonne usinabilité.

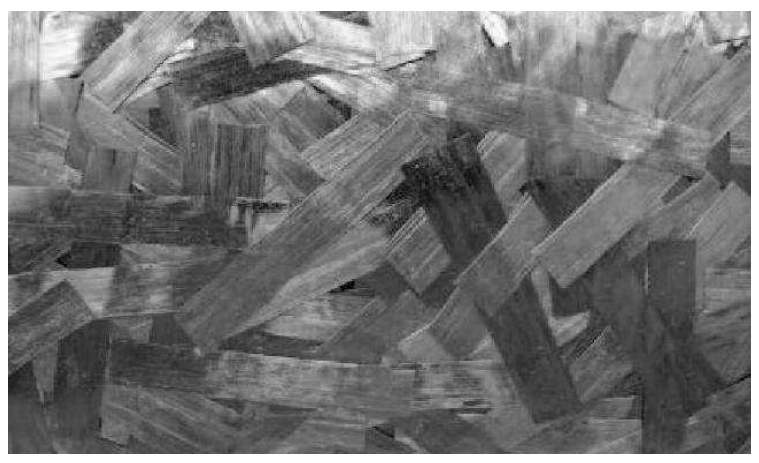

Fig. 1. Vue de surface du pli Hextool non usiné.

Afin de représenter cette mésostructure, une procédure de transition d'échelle en deux étapes est proposée (Fig. 2). Les propriétés mécaniques des renforts unidirectionnels (UD) sont d'abord calculées à partir de celles des fibres et de la résine (un cas largement traité, voir [10]), puis utilisées pour estimer les propriétés macroscopiques du Hextool. On considèrera alors une orientation aléatoire des renforts dans le plan du pli, et on prendra en compte la présence d'une faible portion de résine entre les renforts (5\% en volume). Afin de contourner certaines limites du modèle autocohérent (voir [9]), on considèrera plutôt que les renforts ont une forme de platelet, et non une forme étirée. Ce choix n'influe pas significativement sur les résultats, tout en améliorant fortement la vitesse de calcul (le tenseur d'influence peut être exprimé analytiquement).

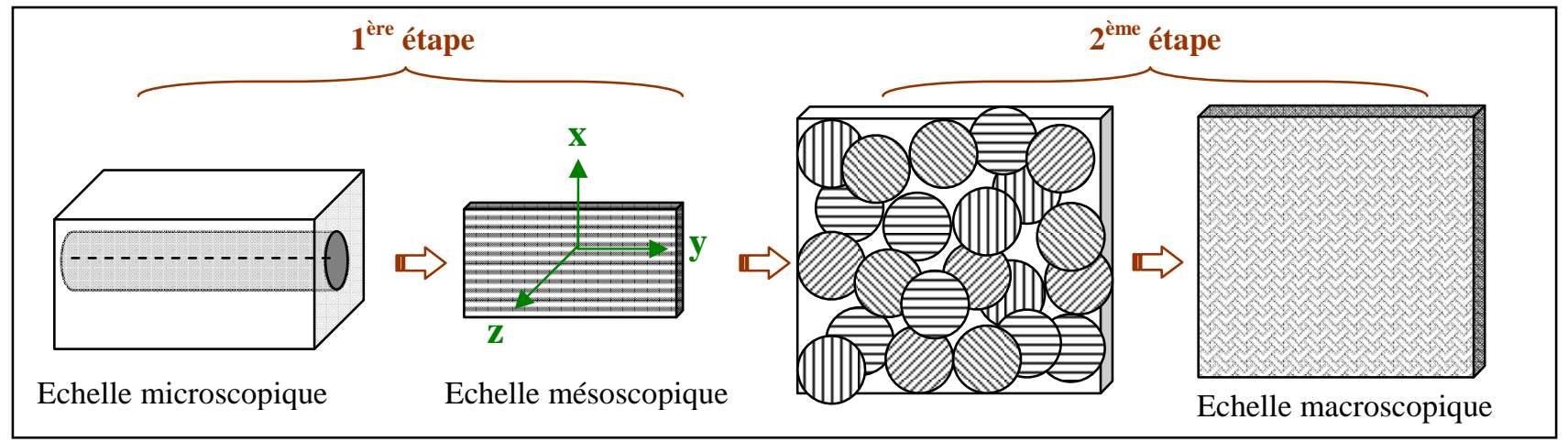

Fig. 2. Méthode de transition d'échelles en deux étapes. 


\subsection{Comportement thermocinétique de la résine}

Afin de décrire correctement l'évolution des propriétés physicochimiques de la résine durant le cycle de fabrication, il est nécessaire de caractériser sa cinétique de réticulation. Dans ce but, une campagne de caractérisation expérimentale a été effectuée par des collaborateurs (voir remerciements), en utilisant la technique de calorimétrie différentielle à balayage (DSC) en mode isotherme et anisotherme. Le degré de cuisson de la résine étant défini comme le ratio de l'enthalpie dégagée sur l'enthalpie totale de réaction, la vitesse de réticulation est exprimée comme une fonction de la température et du degré de cuisson :

$$
\dot{\chi}=\frac{\mathrm{d} \chi}{\mathrm{dt}}=\mathrm{f}(\chi, \mathrm{T})
$$

Différents modèles proposent une description phénoménologique de la cinétique de réticulation. Citons notamment le modèle de Kamal et Sourour [14], très utilisé, ainsi que celui de Bailleul [15]. Ceux-ci proposent une séparation des variables de température et de degré de cuisson, la dépendance à la température étant décrite par une fonction Arrhénienne, et celle au degré de conversion par des lois en puissance (Kamal et Sourour) ou polynomiales (Bailleul). Ici, on utilisera plutôt la méthode «Model Free Kinetics » développée par Vyazovkin et al. [16], qui est supposée permettre une meilleure représentation de la cinétique de réticulation, notamment dans le cas où on combine des essais isothermes et anisothermes. Cette méthode repose sur une interpolation de la cinétique similaire à celle de Bailleul, mais à la différence que l'énergie d'activation $\mathrm{E}_{\mathrm{a}}$, utilisée dans la fonction Arrhénienne de température, devient dépendante du degré de cuisson :

$$
\dot{\chi}=\exp \left(-\frac{\mathrm{E}_{\mathrm{a}}(\chi)}{\mathrm{R} \cdot \mathrm{T}}\right) \cdot \mathrm{P}(\chi),
$$

Une méthode numérique permet alors de déterminer, par optimisation, les valeurs de $\mathrm{E}_{\mathrm{a}}(\chi)$ et $\mathrm{P}(\chi)$ à partir de différents programmes de montée en température. Cette méthode a été appliquée à des essais de montée en température à $5^{\circ} \mathrm{C} / \mathrm{min}, 10^{\circ} \mathrm{C} / \mathrm{min}, 15^{\circ} \mathrm{C} / \mathrm{min}$, et $20^{\circ} \mathrm{C} / \mathrm{min}$, effectués sous atmosphère neutre (azote) avec un appareillage Mettler Toledo DSC1 Star-e System. Les résultats de la simulation ont été confrontés aux résultats expérimentaux pour des isothermes à $190^{\circ} \mathrm{C}$ et $220^{\circ} \mathrm{C}$, avec un relativement bon accord compte tenu des incertitudes expérimentales et des écarts liés à l'interpolation des fonctions. Par ailleurs, les essais anisothermes ont également permis d'évaluer la température de transition vitreuse $\mathrm{T}_{\mathrm{g}}$, qu'on a ensuite paramétrée par l'équation de Di Benedetto.

La cinétique de réticulation ainsi déterminée sur la résine pure a ensuite été appliquée à un cycle de fabrication préconisé par Hexcel [1], en faisant l'approximation que la cinétique du matériau Hextool et celle de la résine pure sont identiques. Le cycle de fabrication consiste en une étape de cuisson de $4 \mathrm{~h}$ à $190^{\circ} \mathrm{C}$, un démoulage de la pièce, suivi d'une post-cuisson sans moule à $220^{\circ} \mathrm{C}$ durant $16 \mathrm{~h}$. Les effets d'inertie thermique ont été négligés dans cette modélisation, comme tous les effets «structurels ». La figure ci-dessous (Fig. 3) récapitule les évolutions de la température, du degré de cuisson et de la température de transition vitreuse, durant le cycle de fabrication. On notera que la résine n'est pas totalement cuite à la fin du cycle à $190^{\circ} \mathrm{C}$, puisque le degré de cuisson est alors de $91,3 \%$. Ceci amène une légère diminution des contraintes par rapport à une résine qui serait totalement cuite, puisque la cuisson est finalisée à $220^{\circ} \mathrm{C}$, où le module élastique de la résine est plus faible et où la relaxation des contraintes est plus importante qu'à $190^{\circ} \mathrm{C}$. 


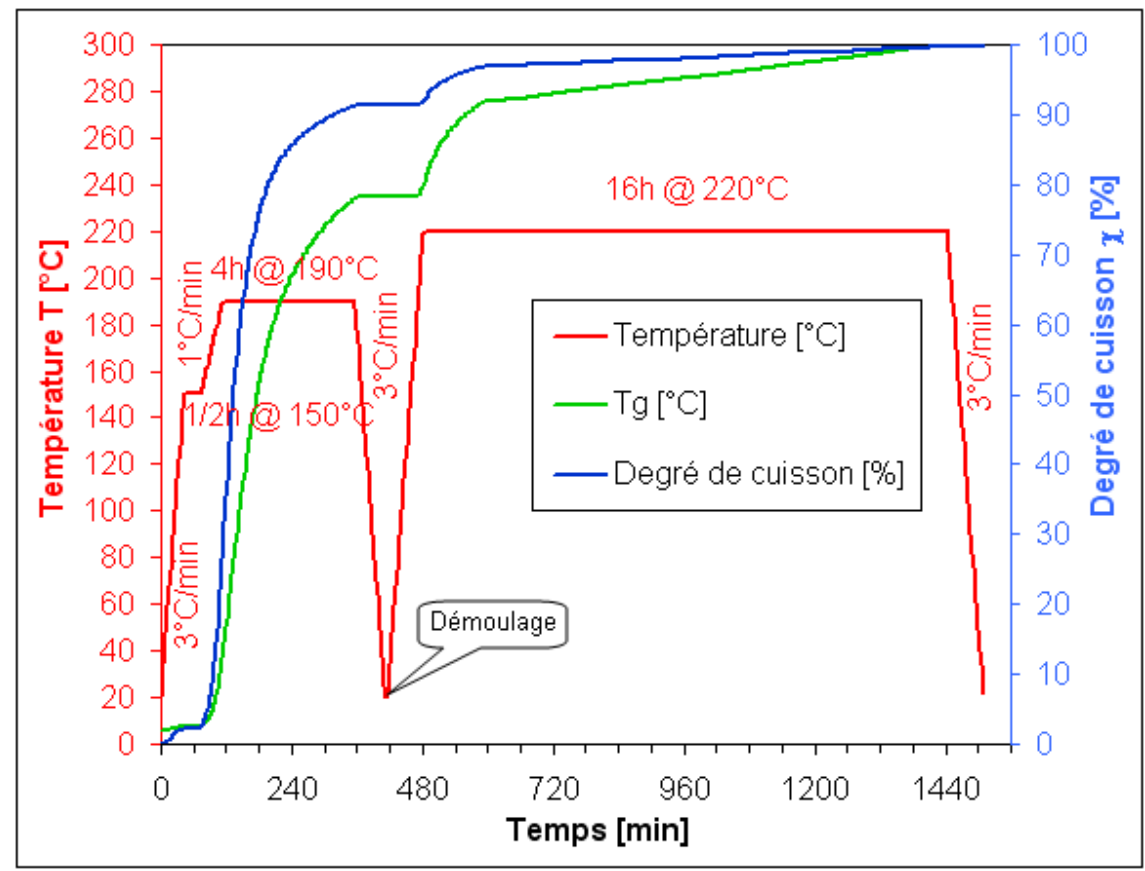

Fig. 3. Cycle de fabrication préconisé pour le Hextool, et thermocinétique de réticulation simulée.

\subsection{Comportement mécanique de la résine}

Les propriétés mécaniques des constituants du Hextool, à température ambiante et à l'état cuit, sont données dans le tableau ci-dessous (Tab. 1). Les modules élastiques de la fibre sont issus de [11], les CDT de la fibre et de la résine nous ont été transmis par Hexcel Composites, et le retrait chimique a été fixé à une valeur typique des résines organiques $(5,7 \%$ de retrait volumique).

\begin{tabular}{|l|ccccc|cc|cc|}
\hline & \multicolumn{4}{|c|}{ Modules mécaniques } & \multicolumn{2}{c|}{ CDT } & \multicolumn{3}{c|}{ CDC } \\
\hline & $\begin{array}{c}\mathrm{E}_{\mathrm{x}} \\
{[\mathrm{GPa}]}\end{array}$ & $\begin{array}{c}\mathrm{E}_{\mathrm{y}}, \mathrm{E}_{\mathrm{z}} \\
{[\mathrm{GPa}]}\end{array}$ & $\begin{array}{c}\mathrm{G}_{\mathrm{yz}} \\
{[\mathrm{GPa}]}\end{array}$ & $\begin{array}{c}\mathrm{G}_{\mathrm{xy}}, \mathrm{G}_{\mathrm{xz}} \\
{[\mathrm{GPa}]}\end{array}$ & $v_{\mathrm{xy}}, v_{\mathrm{xz}}$ & $\begin{array}{c}\alpha_{\mathrm{x}} \\
{\left[10^{-6} / \mathrm{K}\right]}\end{array}$ & $\begin{array}{c}\alpha_{\mathrm{y}}, \alpha_{\mathrm{z}} \\
{\left[10^{-6} / \mathrm{K}\right]}\end{array}$ & $\begin{array}{c}\eta_{\mathrm{x}} \\
{\left[10^{-6}\right]}\end{array}$ & $\begin{array}{c}\eta_{\mathrm{y}}, \eta_{\mathrm{z}} \\
{\left[10^{-6}\right]}\end{array}$ \\
\hline Résine BMI & 3,5 & 3,5 & 1,6 & 1,6 & 0,40 & 38,2 & 38,2 & -19000 & -19000 \\
Fibres AS4 & 207 & 20,7 & 6,9 & 27,6 & 0,20 & $-0,35$ & 11,8 & 0 & 0 \\
\hline
\end{tabular}

Tab. 1. Propriétés mécaniques des constituants à l'état cuit et température ambiante

Afin de décrire correctement le développement des contraintes résiduelles dans le matériau, il est nécessaire de prendre en compte l'évolution des propriétés mécaniques de la résine avec la température T, mais surtout avec le degré de polymérisation $\chi$. En effet, au cours de la cuisson, la résine subit une transformation importante de sa structure physicochimique : elle passe d'un état liquide (avant le point de gel) à un état de solide caoutchoutique, puis de solide vitreux. C'est pourquoi le point de gel est considéré par la plupart des auteurs comme le point à partir duquel la résine acquiert une rigidité (à long terme) non nulle, et commence à stocker des contraintes résiduelles. Le modèle le plus couramment utilisé pour décrire cette évolution de la rhéologie suppose une évolution linéaire du module élastique à partir du point de gel, le coefficient de Poisson étant, lui, supposé constant. Cependant, on lui préfèrera l'expression utilisée par Msallem et al., et issue de la théorie de percolation, qui est bien adaptée aux matériaux polymères $[12,13]$ :

$$
\mathrm{E}(\mathrm{T}, \chi)=\mathrm{E}_{\text {cuit }}(\mathrm{T}) \cdot\left(\frac{\chi^{2}-\chi_{\text {gel }}^{2}}{1-\chi_{\text {gel }}^{2}}\right)^{8 / 3},
$$

où $\chi_{\text {gel }}$ est le degré de conversion au point de gel, et $\mathrm{E}_{\text {cuit }}(\mathrm{T})$ est le module élastique de la résine totalement cuite. L'évolution de ce module à l'état cuit a été déterminée par des collaborateurs par 
des essais mécaniques dynamiques (DMA). La figure ci-dessous (Fig. 4) synthétise les évolutions du module élastique en fonction du degré de cuisson et de la température. Notons que le modèle choisi ici néglige les effets de relaxation à court ou long terme des contraintes dans la résine, et peut donc aboutir à une légère surestimation des contraintes, notamment celles dues au retrait chimique.

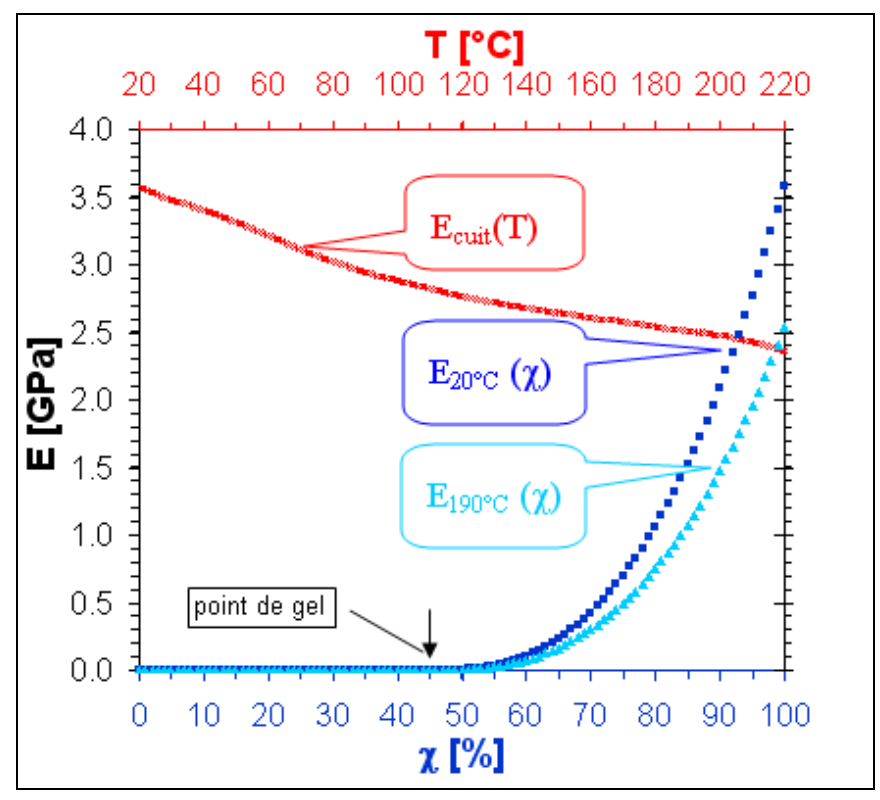

Fig. 4. Evolution du module élastique de la résine avec la température et le degré de cuisson.

Le coefficient de dilatation thermique de la résine subit également des évolutions importantes durant le cycle de cuisson : il augmente avec la température, et diminue avec le degré de cuisson. Ces évolutions ont été décrites via l'interpolation suivante :

$$
\alpha(T, \chi)=\chi \times \alpha_{\text {cuit }}(T)+(1-\chi) \times \alpha_{\text {cru }}(T) \text { avec }\left\{\begin{array}{l}
\alpha_{\text {cuit }}(T)=35.465+0.1191 \times \mathrm{T}+0.0003 \times \mathrm{T}^{2} \\
\alpha_{\text {cru }}(\mathrm{T})=4 \times \alpha_{\text {cuit }}(\mathrm{T})
\end{array}\right.
$$

L'interpolation du CDT de la résine à l'état cuit a été effectuée à l'aide de données fournies par Hexcel Composites sur une gamme de température allant de $20^{\circ} \mathrm{C}$ à $170^{\circ} \mathrm{C}$. L'influence du degré de cuisson sur le CDT est déterminée arbitrairement, mais n'influe pas de façon importante sur les résultats : en effet, les contraintes thermiques sont crées essentiellement à la fin de la post-cuisson, quand la résine est totalement cuite.

\section{Résultats}

\subsection{Propriétés effectives}

Le modèle autocohérent a été utilisé afin de calculer les propriétés mécaniques du Hextool, suivant la méthode décrite plus haut. Les propriétés calculées et mesurées, à l'état cuit et température ambiante sont données dans le tableau ci-dessous (Tab. 2). Le modèle est en assez bon accord avec les résultats expérimentaux (fournis par Hexcel), les écarts étant imputables à l'ondulation naturelle des renforts, ainsi qu'à des problèmes de taille et de représentativité des éprouvettes.

\begin{tabular}{|l|ccccc|cc|cc|}
\hline & \multicolumn{5}{|c|}{ Modules mécaniques } & \multicolumn{2}{c|}{ CDT } & \multicolumn{2}{c|}{ CDC } \\
& $\mathrm{E}_{1}, \mathrm{E}_{2}$ & $\mathrm{E}_{3}$ & $\mathrm{G}_{12}$ & $\mathrm{G}_{13}, \mathrm{G}_{23}$ & $v_{13}, v_{23}$ & $\alpha_{1}, \alpha_{2}$ & $\alpha_{3}$ & $\eta_{1}, \eta_{2}$ & $\eta_{3}$ \\
& {$[\mathrm{GPa}]$} & {$[\mathrm{GPa}]$} & {$[\mathrm{GPa}]$} & {$[\mathrm{GPa}]$} & & {$\left[10^{-6} / \mathrm{K}\right]$} & {$\left[10^{-6} / \mathrm{K}\right]$} & {$\left[10^{-6}\right]$} & {$\left[10^{-6}\right]$} \\
\hline Calculées & 46,1 & 12,1 & 18,1 & 3,8 & 0,37 & 2,98 & 42,5 & -1323 & -15894 \\
Mesurées & $41 \sim 46 \pm 3,8$ & $\mathrm{X}$ & $14,4 \pm 1,8$ & $\mathrm{X}$ & $\mathrm{X}$ & $3,5 \sim 5 \pm 1$ & $45 \sim 52 \pm 1$ & $\mathrm{X}$ & $\mathrm{X}$ \\
\hline
\end{tabular}

Tab. 2. Propriétés mécaniques du Hextol, mesurées et calculées par le modèle autocohérent. 
Les évolutions des propriétés mécaniques de la résine M61 et du Hextool durant la fabrication sont récapitulées sur la figure ci-dessous (Fig. 5). On observe un saut de propriétés au point de gel, puis une évolution importante avec le durcissement de la résine, et durant les phases de refroidissement. La rigidité du matériau dans le plan dépend principalement de celle des fibres et évolue donc assez peu, contrairement aux autres propriétés qui sont pilotées par la résine. Cet effet est encore plus visible si on utilise le modèle de Mori-Tanaka au lieu du modèle autocohérent [3].

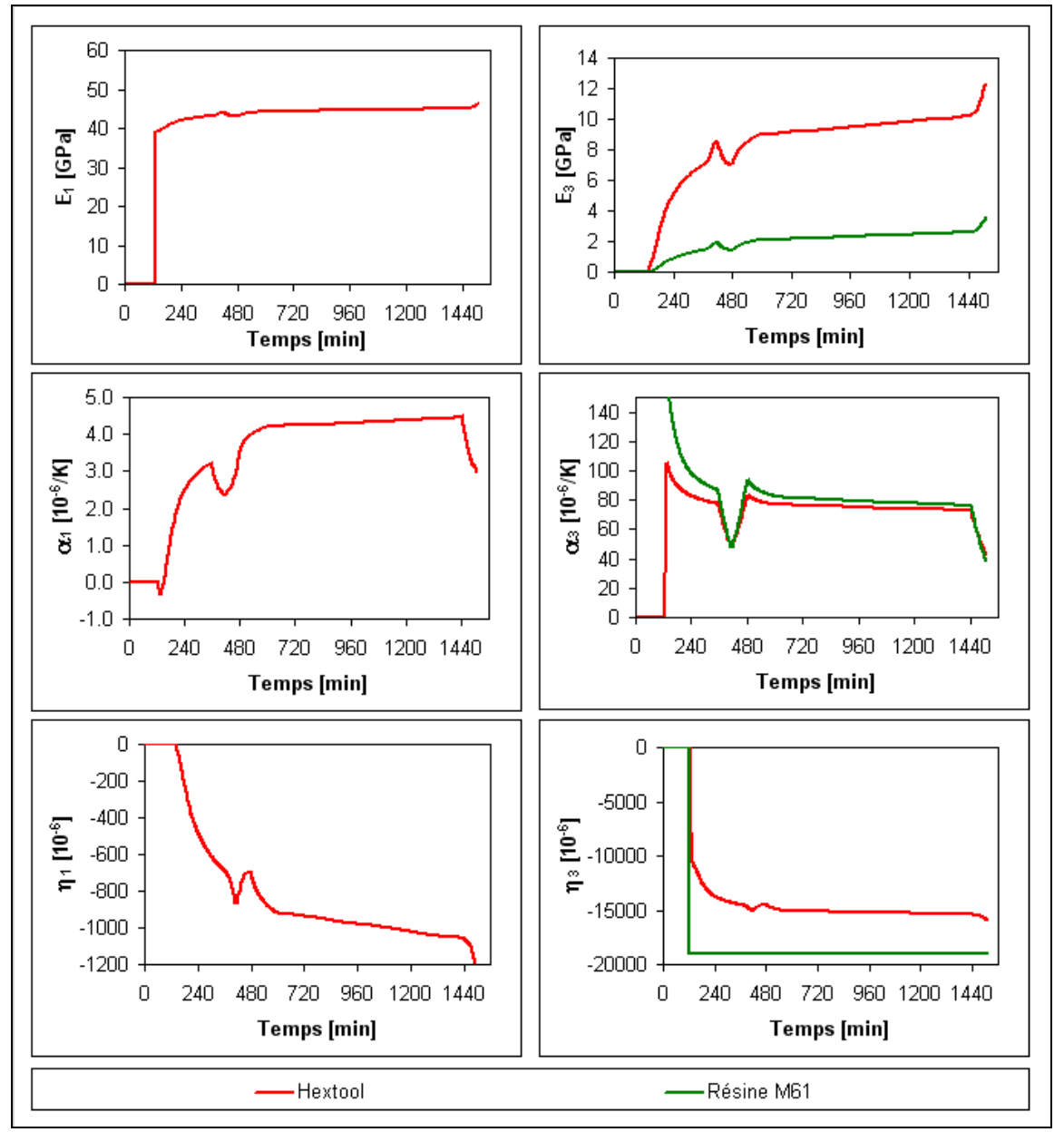

Fig. 5. Evolutions des propriétés mécaniques de la résine M61 et du Hextool durant le procédé de fabrication.

\subsection{Contraintes résiduelles locales}

Les contraintes dans les constituants du matériau ont été calculées de façon incrémentale par le modèle autocohérent d'Eshelby-Kröner, tout au long du cycle de fabrication. Leur évolution est récapitulée dans la figure (Fig. 6), en projection dans le repère local $\mathrm{R}_{\mathrm{xyz}}$ attaché à chaque renfort (voir Fig. 2). Pour simplifier le problème, on a négligé tous les effets structurels et pseudomacroscopiques pouvant apparaître dans une pièce complexe, et on a également considéré le cas d'une cuisson libre de contraintes macroscopiques. On montre, par ailleurs, que ces chargements externes n'influent que très peu sur les contraintes finales.

Les contraintes résiduelles se développent à partir du point de gel, sous l'effet du retrait chimique de la résine, puis durant les phases de refroidissement. Notons que les contraintes thermiques sont beaucoup plus importantes que les contraintes dues au retrait chimique, ce qui souligne l'influence du durcissement de la résine : l'évolution non-linéaire de son module d'Young limite fortement ces contraintes, malgré des retraits thermique et chimique comparables pour la résine pure. Ainsi, à $220^{\circ} \mathrm{C}$, les contraintes dans le matériau sont quasiment nulles.

Le retrait thermochimique de la résine se traduit, dans la direction des fibres (x), par des contraintes de compression dans les éléments rigides (renforts et fibres) et de traction dans la résine. Il entraine 
également des contraintes de traction dans la direction transverse aux fibres (y), et de faibles contraintes à l'échelle microscopique, dans la direction normale au pli (z). Afin de quantifier la tenue de la résine, on a défini un critère de rupture de type Tsaï-Wu en utilisant les contraintes à rupture d'une résine haute résistance [17]. L'inverse du facteur de résistance ainsi obtenu est donné sur la figure (Fig. 6). On constate que sa valeur atteint $63 \%$ à la fin de la fabrication, les contraintes résiduelles diminuent donc considérablement la résistance du matériau. Par ailleurs, le choix du modèle (autocohérent ou Mori-Tanaka) n'influe que très peu sur les contraintes mésoscopiques, mais a en revanche un fort impact sur les contraintes à l'échelle microscopique [3].
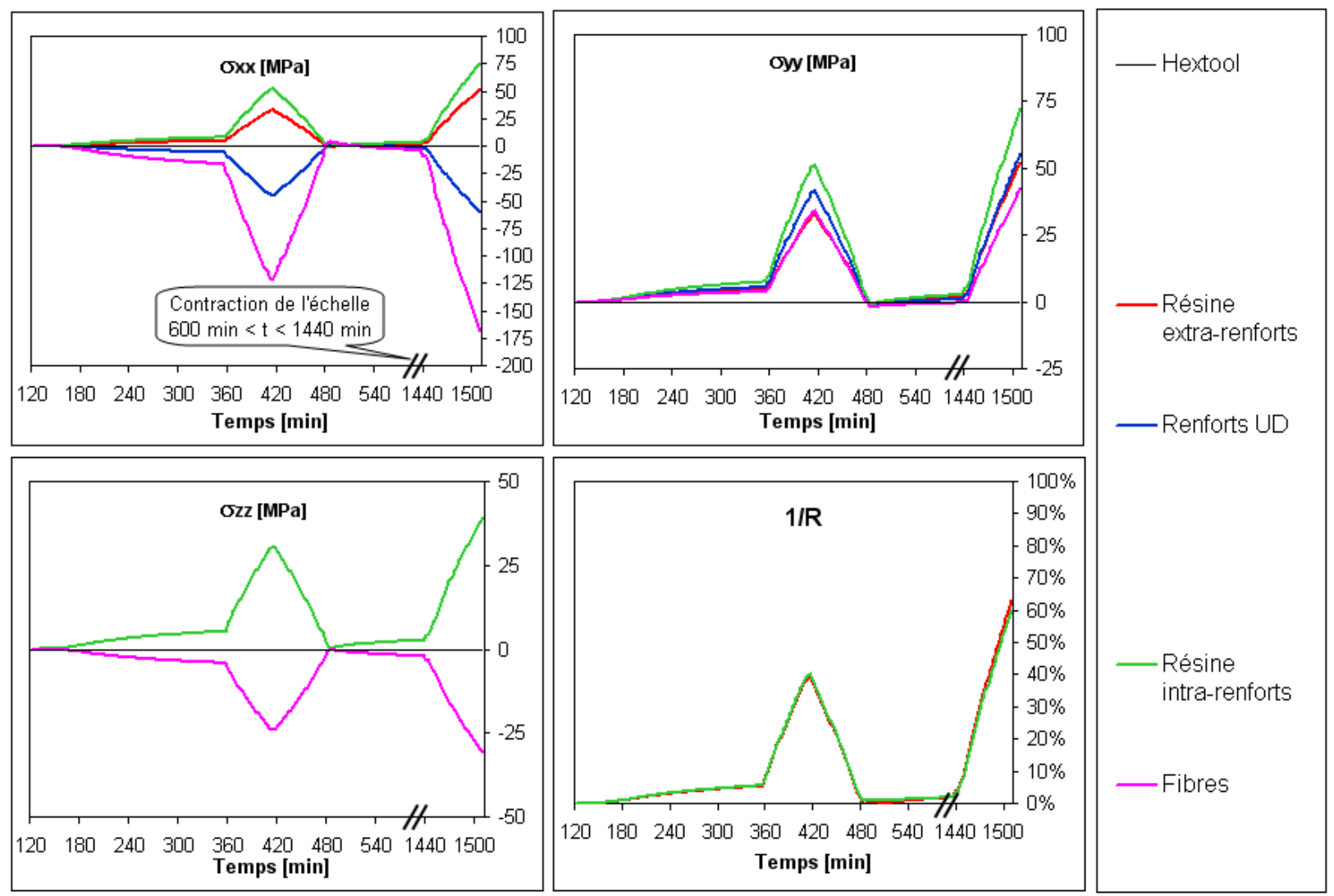

Fig. 6. Evolution des contraintes dans les constituants du Hextool durant le procédé de fabrication.

\subsection{Résistance en traction unidirectionnelle}

Des essais de traction statique effectués par Hexcel Composites ont permis de quantifier la limite à rupture du Hextool en traction unidirectionnelle (260MPa) et en compression (300 MPa). La méthode de transition d'échelles définie plus haut a été confrontée à ces résultats expérimentaux, en calculant les contraintes combinées (résiduelles et de service) dans le matériau, sous chargement uniaxial de -300 MPa à $260 \mathrm{MPa}$. Comme ce chargement ne respecte pas l'isotropie plane du matériau, les contraintes locales dépendent de l'orientation des bandes renforçantes par rapport à l'axe de sollicitation, suivant une évolution $\pi$-sinusoïdale. Dans la figure (Fig. 7) ci-dessous, on a représenté les contraintes $\sigma_{\mathrm{xx}}$ et le facteur $1 / \mathrm{R}$ pour des bandes orientées à $0^{\circ}$ et $90^{\circ}$, qui sont les orientations les plus critiques pour la tenue du matériau.

Les résultats indiquent, pour un chargement de compression, des contraintes faibles dans la résine mais des niveaux de compression importants dans les éléments rigides alignés avec la direction de sollicitation. Ces efforts pourraient expliquer la rupture du matériau en compression, par flambement puis délaminage de ces renforts, ce mécanisme étant en outre favorisé par l'ondulation initiale des renforts (liée à leur placement par superposition aléatoire).

Pour la rupture en traction, les résultats indiquent de forts niveaux de contraintes dans la résine, particulièrement celle contenue dans les orientés à $90^{\circ}$. Ceci tend à indiquer une rupture du 
matériau par la dégradation de ces éléments, même si la valeur du facteur de rupture $1 / \mathrm{R}$ reste inférieure à $90 \%$ (valeur discutable, car très dépendante de la résistance de la résine, qu'on n'a pas caractérisée). En réalité, on constate que la rupture en traction intervient plutôt par délaminage des renforts, c'est à dire fissuration de la résine extra-renforts. Ce constat souligne les limites de l'approche à champ moyen utilisée ici, qui ne donne que la valeur moyenne (sur tout le V.E.R.) des contraintes dans la résine. Les contraintes de cisaillement qui apparaissent entre des renforts orientés différemment ne sont pas représentées, or elles jouent un rôle important dans ces délaminages. L'information partielle délivrée par l'approche champ moyen devrait donc être complétée par une approche à champ complet.

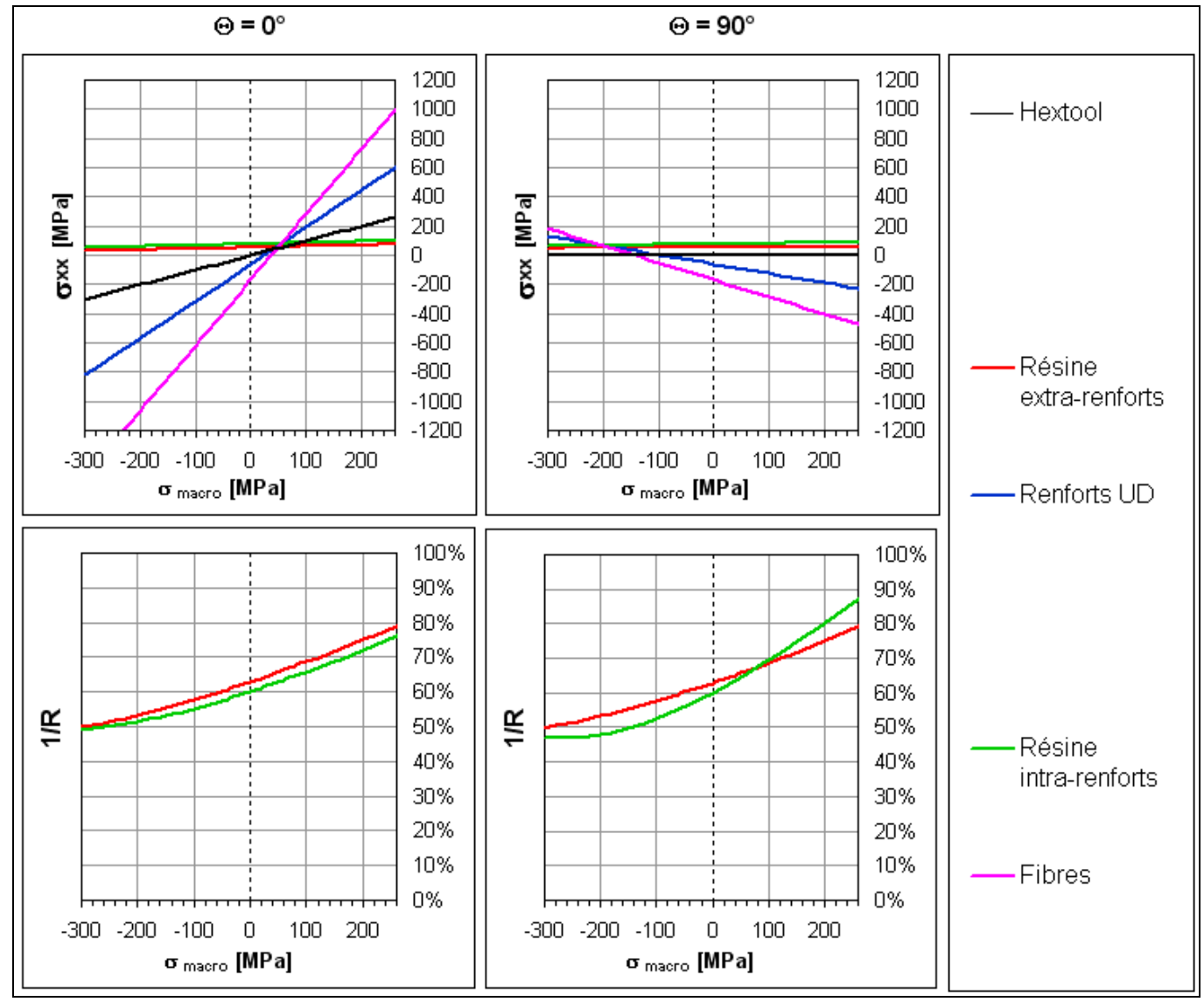

Fig. 7. Contraintes locales calculées pour une sollicitation combinée de fabrication et de chargement uniaxial.

\section{$4 \quad$ Discussion et perspectives}

Dans cet article, une approche de transition d'échelles a été proposée afin d'étudier les contraintes aux échelles locales (mésoscopique et microscopique) dans un matériau composite à renforts mésoscopiques courts. Le développement des contraintes résiduelles de fabrication a été calculé tout au long d'un cycle de fabrication typique, en prenant en compte l'évolution des propriétés mécaniques (rhéologiques et dilatométriques) de la résine, ainsi que sa cinétique de cuisson. Les contraintes générées par une sollicitation de traction unidirectionnelle ont ensuite été ajoutées aux contraintes résiduelles de fabrication. Les résultats nous mènent aux conclusions suivantes :

1. Les contraintes résiduelles de fabrication sont principalement d'origine thermique, même si le retrait chimique de la résine joue aussi un rôle significatif. Par ailleurs, l'intensité de ces dernières dépend très fortement de l'évolution de la rhéologie de la résine durant la cuisson. En outre, on peut estimer que les contraintes résiduelles sont quasiment nulles à environ $220^{\circ} \mathrm{C}$.

2. Les contraintes résiduelles jouent un rôle primordial dans la tenue du matériau, et permettent d'expliquer certains mécanismes de dégradation du matériau. Ces résultats sont donc une première étape vers la simulation de l'endommagement du matériau sous sollicitations 
cycliques. Toutefois, l'approche utilisée ici ne permet pas d'expliquer correctement le comportement à rupture du matériau, pour plusieurs raisons: la méconnaissance du comportement à rupture de la résine, et surtout l'apparition de mécanismes de dégradation par flambement et délaminage des renforts.

Dans des travaux ultérieurs, il serait pertinent d'utiliser une approche à champ complet pour mesurer ces contraintes locales, soit expérimentalement (microtomographie ou corrélation d'images), soit par la simulation du V.E.R. avec les éléments finis. Par ailleurs, ces travaux permettent également d'alimenter en propriétés mécaniques des calculs aux éléments finis, par exemple si l'on s'intéresse aux déformations résiduelles de fabrication sur des structures de grande taille. L'évolution des propriétés du Hextool joue un rôle important dans les interactions moulepièce et pourrait, en outre, induire une dépendance entre la déformée finale de la pièce et les chargement subis durant la fabrication.

\section{Remerciements}

Les auteurs souhaitent remercier C. Dauphin et M. Bonnafoux, ingénieurs chez Hexcel Composites France, pour leur collaboration et pour toutes les informations sur le Hextool qu'ils nous ont procurées. Ils sont aussi particulièrement reconnaissants à K. Szymanska, S. Terekhina et M. Salvia, du Laboratoire de Tribologie et de Dynamique des Systèmes (LTDS, UMR 5513), qui nous ont fourni leurs résultats d'essais mécaniques (DMA) et thermocinétiques (DSC) sur la résine bismaléimide.

\section{Références}

[1] Hexcel Composites France, Hextool User Guide et Hextool DataSheet, www.hexcel.com, 2008.

[2] U.F. Kocks, C.N. Tomé, H.R. Wenk, « Texture and anisotropy ». Cambridge University Press, 1998.

[3] E. Lacoste, S. Fréour, F. Jacquemin, «A multi-scale study of residual stresses created during the cure process of a composite tooling material », Materials Science Forum, Vol. 681, pp. 309-314, 2011.

[4] P.P. Parlevliet, H.E.N. Bersee, A. Beukers, "Residual stresses in thermoplastic composites - a study of the literature. Part I: Formation of residual stresses ». Composites Part A, Vol. 37, pp. 1847-1857, 2006.

[5] P.P. Parlevliet, H.E.N. Bersee, A. Beukers. "Residual stresses in thermoplastic composites - a study of the literature. Part II: Experimental techniques ». Composites Part A, Vol. 38, pp. 651-665, 2007.

[6] P.P. Parlevliet, H.E.N. Bersee, A. Beukers, «Residual stresses in thermoplastic composites - a study of the literature. Part III: Effects of thermal residual stresses ». Composites Part A, Vol. 38, pp. 1581-1596, 2007.

[7] R. Hill, «The essential structure of constitutive laws for metals composites and polycrystals ». Journal of the Mechanics and Physics of Solids, Vol. 15, pp. 79-95, 1967.

[8] T. Mori, K. Tanaka, «Average stress in the matrix and average elastic energy of materials with misfitting inclusions ». Acta Metallurgica, Vol. 21, pp. 571-574, 1973.

[9] E. Lacoste, S. Fréour, F. Jacquemin, «On the validity of the self-consistent scale transition model for inclusions with varying morphologies ». Mechanics of Materials, Vol. 42, pp. 218-226, 2010.

[10] S. Fréour, F. Jacquemin, R. Guillén, «Extension of Mori-Tanaka approach to hygroelastic loading of fiberreinforced composites - Comparison with Eshelby-Kröner self-consistent model ». Journal of Reinforced Plastics and Composites, Vol. 25, pp. 1039-1052, 2006.

[11] S.R. White, K.S. Kim, «Process-induced residual stress analysis of AS4/3501-6 composite material ». Mechanics of Composite Materials and Structures, Vol. 5, pp. 153-86, 1998.

[12] Y.A. Msallem, F. Jacquemin, N. Boyard, A. Poitou, D. Delaunay, S. Chatel, «Material characterization and residual stresses simulation during the manufacturing process of epoxy matrix composites ». Composites Part A, Vol. 41, pp. 108-115, 2010.

[13] D.B. Adolf, R.S. Chambers, «Verification of the capability for quantitative stress prediction during epoxy cure », Polymer, Vol. 38, pp. 5481-5490, 1997.

[14] M.R. Kamal, S. Sourour, « Kinetics and thermal characterization of thermosets cure », Polymer Engineering and Science, Vol. 13, pp. 59-64, 1973.

[15] J.L. Bailleul, «Optimisation du cycle de cuisson des pièces épaisses en matériau composite. Application à un préimprégné résine époxyde/fibres de verre ». Thèse de Doctorat, Université de Nantes, 1997.

[16] N. Sbirrazzuoli, S. Vyazovkin, «Learning about epoxy cure mechanisms from isoconversional analysis of DSC data », Thermochimica Acta, Vol. 388 (1-2), pp. 289-298, 2002.

[17] B. Fiedler, M. Hojo, S. Ochiai, K. Schulte, M. Ando, «Failure behavior of an epoxy matrix under different kinds of static loading », Composites Science and Technology, Vol. 61, pp. 1615-1624, 2001. 